\section{Retención de competencias procedimentales en estudiantes de medicina posterior a un entrenamiento mediante un modelo de simulación (EPROBA)}

\author{
ROBERTO GONZÁLEZ L. ${ }^{1,2}$, FELIPE ALARCÓN O. ${ }^{a}$, \\ HÉCTOR MOLINA Z. ${ }^{2}$, MARÍA GARCÍA-HUIDOBRO D. ${ }^{2,3}$, \\ PATRICIO STEVENS M. ${ }^{2,3}$, RODRIGO REYES M. ${ }^{1,2}$,

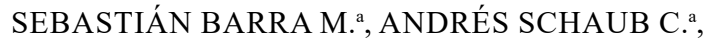

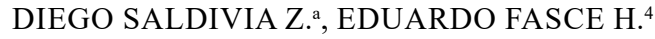

\section{Retention of procedural competences in medicine students after training using a simulation model}

Background: Boot camps are used to acquire skills in simple surgical procedures, such as sutures, in a short period of time. Aim: To assess the retention of the procedural skills of medical students who participated in a simulation-based suture workshop. Material and Methods: One hundred five medical students were trained using the EPROBA methodology (Entrenamiento PROcedimental BAsico), with prior informed consent. The instrument "Objective Structured Assessment Of Technical Skills" (OSATS) was applied prior to and after the workshop, and during retention module, which was carried out between three and four months after the suture workshop. Participants were classified according to the level of previous knowledge in sutures. Results: The students showed significant improvement in their scores after the workshop (improvement from 12.9 to 28.5 points, $p<0.001)$. No significant differences were found between scores after the workshop and those obtained in the retention module (28.5 to 28.1 points, respectively, $p=0.235$ ). No difference in scores were observed when retention was evaluated according to previous knowledge levels. Conclusions: Simulation programs are useful for the acquisition of procedural skills which are retained over time.

(Rev Med Chile 2020; 148: 1427-1434)

Keywords: Education, Medical; Motor Skills; Students, Medical; Simulation training.

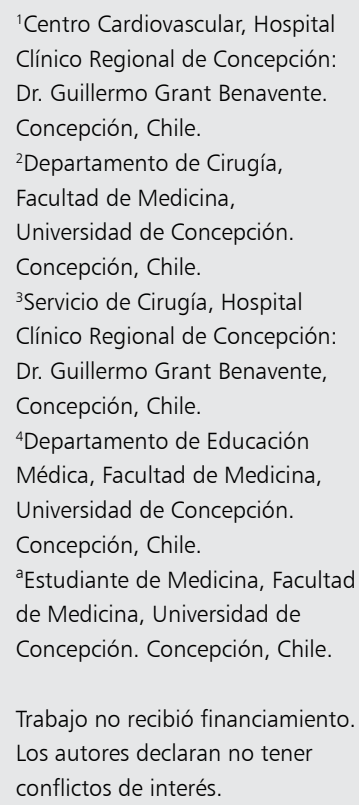

${ }^{1}$ Centro Cardiovascular, Hospital Clínico Regional de Concepción: Dr. Guillermo Grant Benavente. Concepción, Chile. ${ }^{2}$ Departamento de Cirugía, Facultad de Medicina, Universidad de Concepción. Concepción, Chile.

${ }^{3}$ Servicio de Cirugía, Hospital Clínico Regional de Concepción: Dr. Guillermo Grant Benavente, Concepción, Chile.

${ }^{4}$ Departamento de Educación Médica, Facultad de Medicina, Universidad de Concepción. Concepción, Chile.

aEstudiante de Medicina, Facultad de Medicina, Universidad de Concepción. Concepción, Chile.

Trabajo no recibió financiamiento. Los autores declaran no tener conflictos de interés.

Recibido el 24 de abril de 2020 aceptado el 13 de octubre de 2020.

Correspondencia:

Roberto González Lagos Departamento de Cirugía, Facultad de Medicina, Universidad de Concepción, Janequeo esquina Chacabuco S/N, Concepción, Chile. rgonzalezlagos@udec.cl
L

a simulación se ha establecido como una metodología válida para el entrenamiento procedimental en la formación de estudiantes de pregrado y postgrado de medicina ${ }^{1-5}$. Los "campos de entrenamiento", definidos como cursos enfocados en mejorar el aprendizaje, orientación y preparación de los estudiantes que ingresan a un nuevo rol clínico, han sido extensamente descritos ${ }^{6}$. Estas metodologías se han utilizado para la enseñanza de suturas y otros procedimientos, basándose fundamentalmente en el uso de la simulación, la retroalimentación por parte de un docente y el uso de pautas de evaluación cuantitativas estandarizadas ${ }^{7,8}$. 
Estudios han demostrado que es posible enseñar competencias procedimentales básicas en cursos de entrenamiento acotados s,-12 $^{6}$. La mayoría realiza una evaluación previa e inmediatamente posterior a la intervención, logrando objetivamente mejoras significativas entre ambas evaluaciones, sin embargo, una de las limitaciones de dichos modelos es que no aseguran una retención en el tiempo, aspecto relevante al considerar que la implementación de un modelo significa invertir en planificación y el uso de recursos docentes y materiales.

Desde el año 2018, nuestro equipo ha implementado un taller de suturas basado en el modelo EPROBA $^{13}$, el cual se basa en cinco recursos pedagógicos: el entrenamiento mediante simulación clínica, la enseñanza por pares entrenados, evaluación con pautas estandarizadas, enseñanza en pequeños grupos y uso de Tecnologías de Información y Comunicación (TICs).

Nuestro objetivo es evaluar la retención de las competencias procedimentales de estudiantes de medicina que participaron en un taller de sutura de heridas basado en simulación.

\section{Material y Método}

Estudio cuasi-experimental, antes y después. Se implementó un taller de suturas basado en la metodología EPROBA para estudiantes de medicina. Las especificaciones del programa de entrenamiento se detallan en una publicación de nuestro grupo ${ }^{14}$.

\section{Participantes}

Se incluyó a estudiantes de medicina de cuarto año $(\mathrm{n}=105)$ durante la asignatura impartida por el Departamento de Cirugía de la Facultad de Medicina de la Universidad de Concepción, Chile, el año 2019.

La tutoría fue llevada a cabo por estudiantes de medicina previamente formados como "monitores de suturas". El programa de formación de los monitores y su validación se detalla en extensos publicados previamente ${ }^{15,16}$.

\section{Clasificación según conocimientos previos}

Antes del taller, se clasificó el grado de conocimientos en suturas mediante una encuesta que consultó dirigidamente si han recibido instrucción teórico-práctica o han suturado pacientes. Se subdividió la cohorte en tres niveles: Grupo A - ha recibido instrucción teórico-práctica en suturas y ha suturado pacientes; Grupo B - solo instrucción teórico-práctica en modelos de simulación y Grupo C - sin conocimientos previos en suturas.

\section{Taller de suturas}

La metodología EPROBA incluye un programa teórico práctico. El bloque teórico consistió en el uso de TICs, que correspondió a material audiovisual de elaboración propia relativo a los contenidos entrenados en el bloque práctico.

Se usó un modelo biológico de simulación compuesto por una pata de cerdo (cruda, refrigerada y no congelada). Cada participante tuvo a disposición un set de materiales compuesto por una tabla de soporte para el modelo, porta aguja, tijera recta roma aguda y pinza quirúrgica, suturas, seda para ligar vasos, guantes quirúrgicos, jeringas de $10 \mathrm{ml}$, agujas $21 \mathrm{G}$ y ampollas de solución fisiológica de $10 \mathrm{ml}$.

El programa incluyó la tutoría en los aspectos: técnica aséptica, uso de instrumental básico, preparación de campo quirúrgico, infiltración anestésica local, sutura continua, sutura discontinua y nudos manuales.

El bloque práctico se compuso de dos sesiones de cuatro horas académicas, separadas por una semana. Cada sesión estuvo compuesta por una introducción donde se plantearon los objetivos e instrucciones, seguido de un período de práctica con tiempos definidos para cada tarea y una reunión al final de la sesión donde se realizó retroalimentación y se resolvieron dudas.

Los estudiantes se dividieron aleatoriamente en grupos de seis y fueron tutorizados por los monitores de suturas, quienes tuvieron el objetivo de observar, enseñar y retroalimentar a los participantes para mejorar en cada aspecto de los procedimientos.

\section{Evaluación}

Se objetivó el desempeño en la técnica de sutura mediante el instrumento "The Objective Structured Assessment of Technical Skills" (OSATS) ${ }^{17}$, se utilizó la Escala global OSATS constituida en un 
formato Likert de 6 ítems los cuales corresponden a: "manejo de tejidos", "tiempo y motricidad", "manipulación de instrumentos", "uso apropiado del instrumental quirúrgico", "planificación y ritmo" y "conocimiento del procedimiento específico". Cada ítem posee hasta un máximo de cinco puntos, obteniendo un rango de puntajes de 6 a 30 puntos.

Las evaluaciones posteriores al taller de suturas y del módulo de retención fueron realizadas por tutores que no participaron directamente en el entrenamiento del grupo de estudiantes.

\section{Módulo de retención}

La retención de la competencia procedimental fue medida en un tiempo establecido de tres meses como plazo mínimo y cuatro meses como plazo máximo. Este módulo estuvo constituido de forma similar al taller de suturas, con la salvedad de que se estableció un breve período de práctica libre inicial de diez minutos, sin intervención de los monitores, y posteriormente se evaluó un ejercicio de sutura discontinua en modelo biológico con el instrumento OSATS. Luego de la evaluación, se establecieron tiempos de práctica tutorizada en cada tarea abordada durante el entrenamiento, a modo de retroalimentar y resolver dudas.

Mediante una encuesta se consultó dirigidamente si el estudiante realizó procedimientos de sutura de heridas en pacientes durante el intervalo de tiempo transcurrido entre el taller de suturas y el módulo de retención. Por último, al finalizar la intervención se solicitó realizar un comentario de apreciación personal sobre el módulo de retención.

\section{Análisis de la información}

Se compararon los puntajes promedio de la Escala global OSATS usando la prueba de $U$ Mann-Whitney para variables no paramétricas y ANOVA. Se utilizó SPSS versión $25^{\oplus}$ para el análisis estadístico, considerando significativo un valor $\mathrm{p}<0,05$.

\section{Consideraciones éticas}

El estudio e implementación de una intervención tutorizada para la enseñanza de competencias procedimentales básicas fue aprobada por el comité de ética de la Facultad de Medicina de la Universidad de Concepción, Chile. Todos los participantes firmaron consentimiento informado y las actividades fueron realizadas siempre bajo supervisión del autor principal (RGL).

\section{Resultados}

Todos los participantes $(\mathrm{n}=105)$ cumplieron con la asistencia al taller de suturas y al módulo de retención, con un intervalo entre ambas intervenciones de tres meses como mínimo y cuatro meses como máximo (catorce semanas en promedio).

Al clasificar los estudiantes según el nivel de conocimientos previos en suturas, $11(10,5 \%)$ estudiantes pertenecieron al Grupo A, 32 (30,5\%) pertenecieron al Grupo B, y $62(59,0 \%)$ estudiantes al Grupo C.

Los resultados del taller de suturas mostraron que, en promedio, el universo de estudiantes logró un aumento significativo en la calificación de la Escala global OSATS al comparar los puntajes pre taller y post taller (de 12,9 a 28,5 puntos, respectivamente, $\mathrm{p}<0,001)$. Al analizar dichos resultados según el nivel de conocimientos previos, se observó que los puntajes de ingreso al taller en la Escala global OSATS mostraron diferencias entre ellos (18,0, 15,0 y 11,1 puntos, en los grupos A, B y C, respectivamente, $p<0,001)$. Sin embargo, no se observaron diferencias significativas posterior al taller entre dichos grupos $(\mathrm{p}=0,407)$.

$\mathrm{Al}$ evaluar los resultados del módulo de retención, el puntaje de la Escala global OSATS se mantuvo similar en relación al puntaje post taller $(28,5$ puntos en post taller y 28,1 puntos en retención, $p=0,235)$, ver Figura 1. Este resultado se mantuvo en todos los ítems que componen la Escala global OSATS observándose diferencias mínimas entre ambas evaluaciones (Figura 2). Tampoco se encontraron diferencias significativas en los puntajes del módulo de retención según el nivel de conocimiento previo del estudiante (26,9 puntos, 28,8 puntos y 28,1 puntos en los Grupos A, B y C, respectivamente, $\mathrm{p}=0,058$ ) (Figura 3).

Durante el período entre el taller y el módulo de retención $12(11,4 \%)$ estudiantes realizaron suturas en pacientes durante su asistencia a turnos voluntarios en servicio de urgencia, con una mediana de tres procedimientos.

Se seleccionaron comentarios de parte de los estudiantes al finalizar la intervención, los cuales se describen en la Tabla 1. 


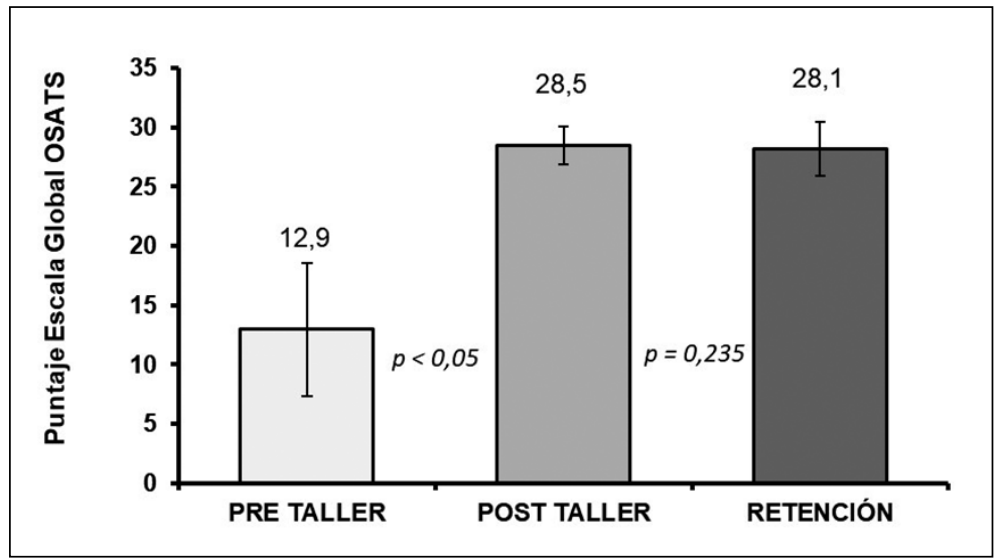

Figura 1. Puntajes promedio de la Escala global OSATS previo al taller, posterior al taller y del módulo de retención. OSATS: The Objective Structured Assessment Of Technical Skills.

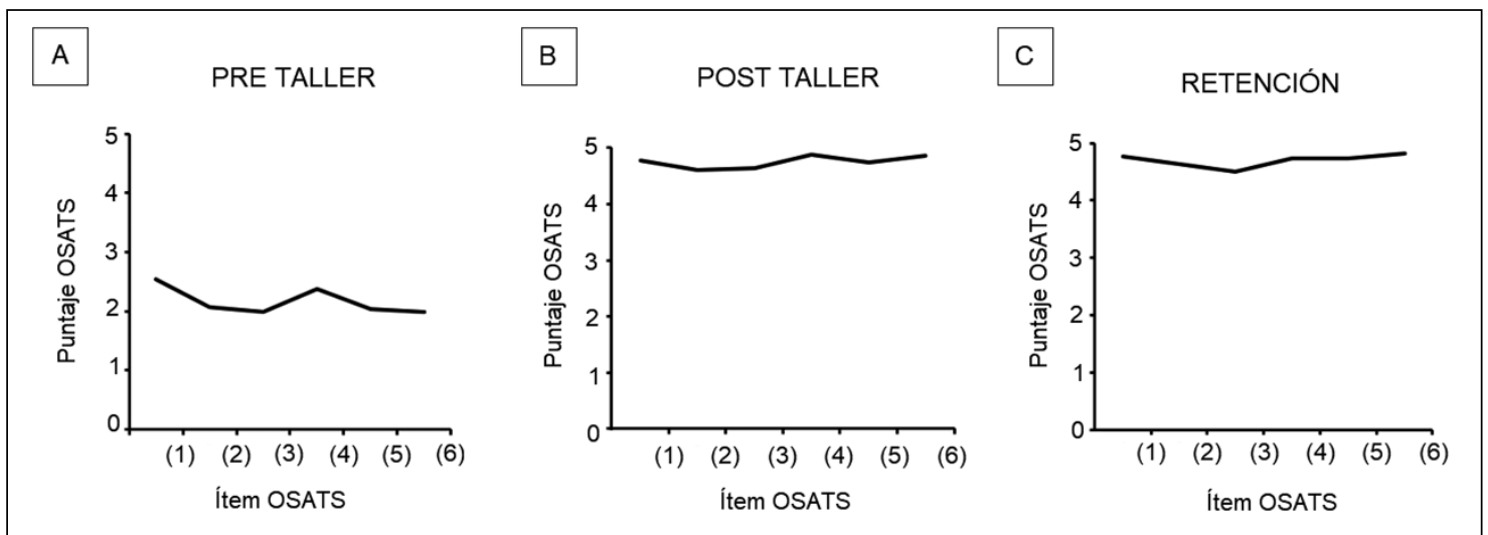

Figura 2. Resultados Escala global OSATS según ítems. 1A. Previo al taller. 2B. Posterior al taller. 2C. Módulo de retención. OSATS: The Objective Structured Assessment Of Technical Skills; (1): Manejo de tejidos; (2): Tiempo y motricidad; (3): Manipulación de instrumentos; (4): Uso apropiado del instrumental quirúrgico; (5): Planificación y ritmo; (6): Conocimiento del procedimiento específico.

Tabla 1. Comentarios seleccionados de algunos participantes al finalizar el módulo de retención

Comentario 1: "No estaba segura si recordaba cómo suturar, y al ir practicando me di cuenta que sí. Me gustó este taller de retención porque si en algún momento tengo que suturar, sé que el procedimiento está en mi memoria"

Comentario 2: "Al principio me costó poner los primeros puntos, pero luego de 3 o 4 intentos logré "soltar la mano". Me alegra saber que recordé todo lo aprendido en sesiones anteriores"

Comentario 3: "El módulo de retención fue muy útil. Ayuda a tener confianza en que se logra desarrollar las destrezas básicas, además de ajustar detalles"

\section{Discusión}

Nuestra experiencia demostró que los estudiantes intervenidos mediante un taller de sutura de heridas basado en la metodología EPROBA mantienen el nivel de competencia procedimental adquirido meses posterior al entrenamiento inicial, sin mostrar diferencias significativas en la evaluación mediante un instrumento de evaluación estandarizada. Además, la retención de competencias procedimentales fue similar entre los grupos, independiente de su nivel de conocimiento previo en suturas. 


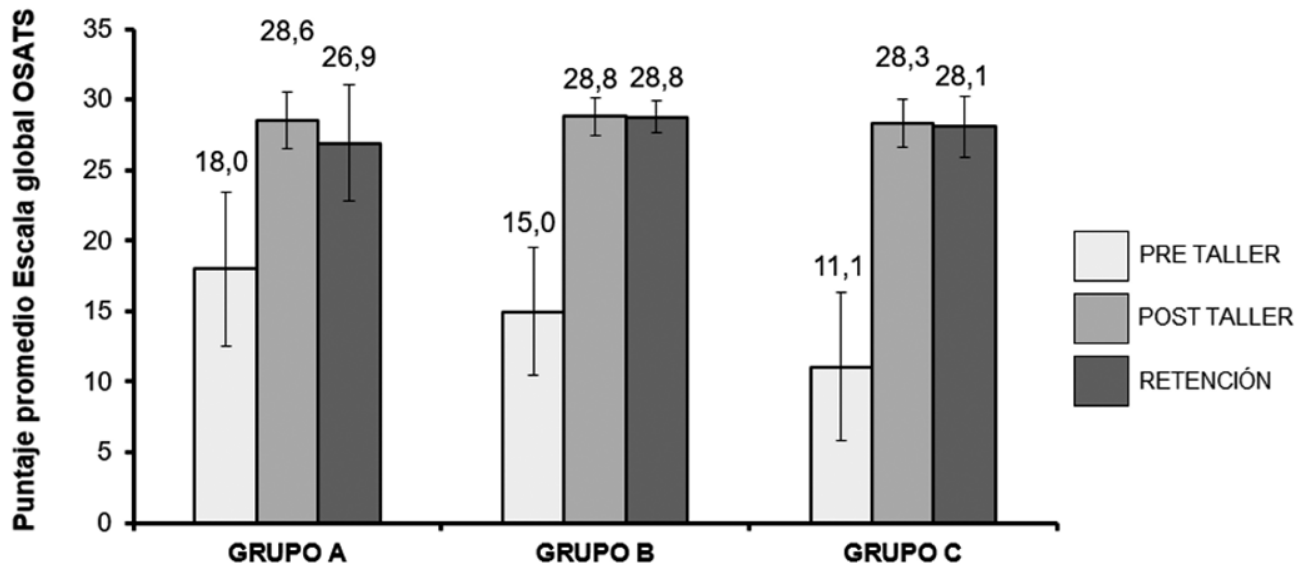

Figura 3. Resultados escala global OSATS según experiencia, previo al taller, posterior al taller y del módulo de retención. OSATS: The Objective Structured Assessment Of Technical Skills. Experiencia del estudiante: Grupo A - ha recibido instrucción teórico práctica en suturas y ha suturado pacientes; Grupo B - solo instrucción teórico-práctica en modelos de simulación; Grupo C - sin conocimientos previos en suturas. Existen diferencias significativas al comparar los puntajes previos al taller según la experiencia del estudiante $(p<0,001)$. No hay diferencias significativas entre los puntajes posteriores al taller según grupo de experiencia $(p=0,407)$. No hay diferencias significativas entre los puntajes del módulo de retención según grupos de experiencia $(p=0,058)$.

Experiencias similares que evaluaron la retención de competencias en programas basados en simulación han reportado resultados contradictorios. Weiss y cols. ${ }^{18}$, en un curso intensivo de tres días de entrenamiento que incluyó a internos de la carrera de medicina, reportaron una disminución significativa de la competencia quirúrgica luego de tres meses de la intervención. Kahol y cols. ${ }^{19}$, realizaron un entrenamiento basado en simulación orientado a competencias laparoscópicas básicas, y evaluaron la retención cada mes durante seis meses, observando una disminución significativa de la competencia quirúrgica después del cuarto mes.

En contraparte, Green y cols. ${ }^{20}$, realizaron un curso de entrenamiento de competencias procedimentales básicas durante los últimos meses de pregrado de medicina y determinaron la retención después de aproximadamente tres meses en quienes ingresaron a la residencia de cirugía general, observando no solo la retención de las competencias, sino también puntajes significativamente mayores en comparación con los participantes que no asistieron a dicho curso en pregrado. Sigh y cols. ${ }^{21}$, realizaron una intervención de simulación "inmersiva" de una semana para internos de me- dicina, observando una mejoría persistente luego de seis meses de intervención en sus habilidades clínicas, procedimentales y en su autopercepción, en comparación con sus compañeros no participantes.

Los campos de entrenamiento, en su mayoría, se basan en las teorías de la adquisición del aprendizaje motor, tales como la práctica deliberada, la disponibilidad de la asistencia de un experto, el feedback continuo y la práctica en grupos pequeños ${ }^{22}$, sin embargo, existe una clara falta de estandarización entre dichos programas, difiriendo, entre otras cosas, en su modelo de simulación, duración y espaciamiento de las prácticas, uso de docentes o estudiantes pares y resultados estudiados. Esta heterogeneidad dificulta la comparación de los resultados, por lo que el uso de indicadores objetivos, tales como pautas de evaluación estandarizadas, se ha propuesto como una necesidad para el análisis estadístico de la evidencia disponible ${ }^{23}$.

En nuestro estudio, la evaluación se centró en un procedimiento, la sutura de heridas. Esto difiere de otras experiencias donde se evaluaron, además, diferentes tipos de puntos discontinuos, sutura continua y tipos de nudos quirúrgicos, que, debido a ser entrenadas en un período acotado, 
puede resultar en una reducción de los períodos de práctica y la atención que se le dedica a cada tarea, pudiendo afectar la consolidación de estas competencias ${ }^{18,19}$. La necesidad de práctica se evidencia en estudios que han demostrado que, para mantener un patrón de desempeño constante, el estudiante debe practicar al menos 6 a 11 veces un mismo procedimiento ${ }^{24}$.

En nuestro caso, la selección del intervalo de tiempo entre el taller y el módulo de retención se basó en el intervalo definido por Weiss y cols. ${ }^{18}$ quienes mediante una experiencia de evaluación de la retención a tres meses sostuvieron que los campos de entrenamiento no aseguran la retención de las competencias. Además, se evitaron intervalos mayores de modo que, al momento de la medición, los estudiantes hubiesen tenido el mínimo de experiencias en escenarios clínicos reales, tales como turnos voluntarios, tutoría por cirujanos docentes durante sus prácticas hospitalarias o la asistencia a talleres organizados por congresos de estudiantes, de modo de que dichas experiencias no alteren los resultados de la retención, logrando una evaluación más fidedigna.

El bajo nivel de exposición a estos procedimientos en el grupo de estudio seleccionado (estudiantes de medicina de cuarto año) puede ser considerado una fortaleza al analizar nuestros resultados. Cabe mencionar que experiencias similares se realizaron con internos de Cirugía quienes, por características propias de los diferentes campos clínicos, reportaron participar en un número sustancialmente mayor de procedimientos quirúrgicos ${ }^{21}$.

El nivel de experiencia de los estudiantes es un aspecto para considerar al momento de evaluar la competencia procedimental. Estudiantes que habían realizado un mayor número de procedimientos a priori al taller poseían mejores resultados al comienzo de la intervención, sin embargo, esto no incidió significativamente en los puntajes post taller ni de retención. Este resultado coincide con experiencias anteriores ${ }^{25-27}$, aludiendo a que un programa estructurado es capaz de homogeneizar la competencia procedimental de los estudiantes.

La autopercepción sobre la competencia procedimental es un factor importante a considerar más allá de la evaluación externa. Los estudiantes expuestos a campos de entrenamiento tienen menores niveles de estrés y ansiedad al momento de enfrentarse a procedimientos en el ámbito clínico, lo que los hace más ávidos a exponerse a dichas situaciones y les permite centrarse en el aprendizaje de procedimientos más complejos, siendo un aspecto relevante independientemente del nivel de retención de las competencias ${ }^{28-30}$. Cabe destacar que Nakazato y cols. ${ }^{31}$, reportaron que una intervención de un solo día para residentes de cirugía es capaz de mejorar significativamente la confianza del residente en competencias procedimentales básicas.

El modelo implementado ha sido bien evaluado por los estudiantes ${ }^{14}$, además, el módulo de retención ha sido valorado como una instancia para consolidar lo aprendido, esto hace plantear la posibilidad de mantener el módulo de retención dentro del programa, debido a sus beneficios.

Varias limitaciones en nuestro estudio pueden ser discutidas. La ausencia de un grupo de control limita la validez externa y puede provocar un sesgo de confusión, lo que dificulta la asociación de la intervención con los resultados obtenidos. Además, los estudiantes están expuestos a la "reactividad del participante" 32 concepto comúnmente denominado el "efecto Hawthorne", ya que conocen el momento y el tipo de ejercicio con los que serán evaluados, pudiendo focalizar su interés y práctica principalmente en dicho procedimiento. Por último, nuestro estudio realiza una medición de la retención en un período único. A pesar de que experiencias han reportado un límite tras el cual se observó un deterioro significativo de la competencia procedimental, es probable que dicho período varíe según el modelo implementado, debido a los múltiples factores que inciden en la adquisición y retención de las competencias procedimentales ${ }^{22}$. De este modo, conocer el comportamiento de la retención de un programa de entrenamiento permite identificar y seleccionar las estrategias más exitosas.

A pesar de las limitaciones mencionadas, se presenta una metodología reproducible en nuestro medio, relativamente de bajo costo y que potencialmente puede entregar competencias en sutura en un período corto de entrenamiento y mantener dicha competencia en el tiempo. De este modo, dado que la sutura de heridas es solo una de las competencias procedimentales que debe adquirir el estudiante de medicina, este modelo puede ser modificado, complejizado, ser aplicado a otros procedimientos o también, ser incluido en la formación de otros profesionales del área 
de la salud como de distintas áreas que requieran la adquisición de competencias procedimentales, de acuerdo con los objetivos curriculares de cada institución.

En conclusión, nuestra experiencia demuestra que un programa de simulación es útil para la adquisición de competencias procedimentales quirúrgicas básicas, logrando una mejora significativa y mantenida en el tiempo.

\section{Referencias}

1. Corvetto M, Bravo MP, Montaña R, Utili F, Escudero E, Boza C, et al. Simulación en educación médica: una sinopsis. Rev Med Chile 2013; 141: 70-9.

2. Selzer D. Overview of Simulation in Surgery. In: Stefanidis D, Korndorffer J, Sweet R. Editors, Comprehensive Healthcare Simulation: Surgery and Surgical Subspecialties. USA: Springer; 2019.p.13-23.

3. Sadideen H, Kneebone R. Practical skills teaching in contemporary surgical education: how can educational theory be applied to promote effective learning? Am J Surg 2012; 204: 396-401.

4. Owen H. Early use of simulation in medical education. Simul Healthc 2012; 7: 102-16.

5. Moya P, Ruz M, Parraguez E, Carreño V, Rodríguez A, Froes P. Efectividad de la simulación en la educación médica desde la perspectiva de seguridad de pacientes. Rev Med Chile 2017; 145: 514-26.

6. Blackmore C, Austin J, Lopushinky SR, Donnon T. Effects of postgraduate medical education "boot camps" on clinical skills, knowledge, and confidence: a meta-analysis. J Grad Med Educ 2014; 6: 643-52.

7. Xeroulis G, Park J, Moulton C, Reznick R, Leblanc V, Dubrowski A. Teaching suturing and knot-tying skills to medical students: a randomized controlled study comparing computer-based video instruction and (concurrent and summary) expert feedback. Surgery 2007; 141: 442-9.

8. Porte M, Xeroulis G, Reznick R, Dubrowski A. Verbal feedback from an expert is more effective than self-accessed feedback about motion efficiency in learning new surgical skills. Am J Surg 2007; 193: 105-10.

9. Sonnadara R, Van Vliet A, Safir O, Alman B, Ferguson P, Kraemer W, et al. Orthopedic boot camp: Examining the effectiveness of an intensive surgical skills course. Surgery 2011; 149: 745-9.

10. Bamford R, Langdon L, Rodd C, Eastaugh-Waring S, Coulston J. Core Trainee Boot Camp, a Method for Improving Technical and Non-Technical Skills of Novice
Surgical Trainees. A Before and After Study. Int J Surg 2018; 57: 60-5.

11. Gershuni V, Woodhause J, Brunt L. Retention of suturing and knot-tying skills in senior medical students after proficiency-based training: Results of a prospective, randomized trial. Surgery 2013; 154: 823-30.

12. Bevilacqua L, Simon J, Rutigliano D, Sorrento J, Wackett A, Chandran L, et al. Surgical Boot Camp for Fourth-Year Medical Students: Impact on Objective Skills and Subjective Confidence. Surgery 2020; 167: 298-301.

13. González R. Metodología de un modelo de entrenamiento en competencias procedimentales quirúrgicas básicas: EPROBA (Entrenamiento procedimental básico). 1st ed. Concepción (Chile): Editorial Universidad de Concepción; 2019. 67p.

14. González R, Molina H, García-Huidobro M, Stevens P, Reyes R, Alarcón F, et al. Entrenamiento de competencias procedimentales quirúrgicas básicas en estudiantes de medicina mediante un modelo de simulación (EPROBA). Rev Cir 2020; 72: 523-9.

15. González R, Molina H, García-Huidobro M, Stevens P, Jadue A, Riquelme A, et al. Implementación de taller de monitores de sutura en alumnos de pregrado de medicina. Rev Cir 2019; 71: 122-8.

16. González R, Molina H, García-Huidobro M, Stevens $\mathrm{P}$, Jadue A, Riquelme A, et al. Basic suture techniques for medicine students: comparative results according to training by surgeons versus peers. Cir Cir 2019; 87: 624-9.

17. Martin J, Regehr G, Reznick R, MacRae H, Mur-naghan J, Hutchison C, et al. Objective structured assessment of technical skill (OSATS) for surgical residents. Br J Surg 1997; 84: 273-8.

18. Weiss J, Farr D, Abdelfattah K, Hogg D, Scott D. A proficiency-based surgical boot camp may not provide trainees with a durable foundation in fundamental surgical skills. Am J Surg 2019; 217: 244-9.

19. Kahol K, Ashby A, Smith M, Ferrara J. Quantitative evaluation of retention of surgical skills learned in simulation. J Surg 2010; 67:421-6.

20. Green C, Huang E, Zhae N, O’Sullivan P, Kim E, Chern $\mathrm{H}$. Technical skill improvement with surgical preparatory courses: What advantages are reflected in residency? Am J Surg 2017; 216: 155-9.

21. Sigh P, Aggarwal R, Pucher P, Hashimoto D, Middleton $\mathrm{K}$, Beyer-Berjot L, et al. An inmersive "simulation week" enhances clinical performance of incoming surgical interns improved performance persist at 6 months follow up. Surgery 2015;157: 432-3.

22. Salideen H, Kneebone R. Practical skills teaching in 
contemporary surgical education: how can educational theory be applied to promote effective learning? Am J Surg 2012; 204: 396-401.

23. Berner J, Ewertz E. Bases teóricas del uso simulación para el entrenamiento en cirugía. Rev Chil Cir 2018; 70: 382-8.

24. Kozmenko V, Kozmenko L, Schellpffefer Sh, Kay A. Theor Kozmenko. Theory and Practice of Developing an Effective Simulation Curriculum. Clinical Simulation 2019; 207-15.

25. González R, Molina H, García-Huidobro M, Stevens $\mathrm{P}$, Reyes R, Barra S, et al. Rol de conocimientos previos en los resultados de un programa de entrenamiento en sutura de heridas para estudiantes de medicina mediante metodología EPROBA. Rev Cir 2021; 73. Disponible en: DOI:10.35687/s2452-45492021002750.

26. Martin AN, Hu Y, Le YA, Brooks KD, Mahmutovic A, Choi J, et al. Predicting surgical skill acquisition in preclinical medical students. Am J Surg 2016; 212: 596-601.

27. Hughes DT, Forest SJ, Foitl R, Chao E. Influence of medical students' past experiences and innate dexte- rity on suturing performance. Am J Surg 2014; 208 : 302-6.

28. Torres C, Goïty L, Muñoz N, Drago P. Entrustable Professional Activities: Una propuesta innovadora para la evaluación de competencias médicas. Rev Med Chile 2018; 146: 1064-9.

29. Radcliffe C, Lester H. Perceived stress during undergraduate medical training: A qualitative study. Med Educ 2003; 37: 32-8.

30. Parent R, Plerhoples T, Long E, Zimmer D, Teshome M, Mohr C, et al. Early, Intermediate, and Late Effects of a Surgical Skills "Boot Camp" on an Objective Structured Assessment of Technical Skills: A Randomized Controlled Study. J Am Coll Surg 2010; 210: 984-9.

31. Nakazato T, Callahan Z, Kuchta Z, Linn J, Joehl R, Ujiki M. 1-day simulation-based boot camp for incoming general surgery residents improves confidence and technical skills. Surgery 2019;166: 572-9.

32. Paradis E, Sutkin G. Beyond a good story: from Hawthorne Effect to reactivity in health professions education research. Med Educ 2017; 51: 31-9. 